\title{
Antibiotic Induced Cutaneous Rash in Infectious Mononucleosis: Overview of the Literature
}

\author{
Katinka Ónodi-Nagy ${ }^{1 *}$, Zsuzsanna Bata-Csörgő ${ }^{1}$, Erika Varga ${ }^{1}$, Lajos Kemény ${ }^{1}$ and Ágnes Kinyó ${ }^{1,2}$ \\ ${ }^{1}$ Department of Dermatology and Allergology, University of Szeged, Albert Szent-Györgyi Medical Center, Hungary \\ ${ }^{2}$ Department of Dermatology, Venereology and Oncodermatology, University of Pécs, Hungary
}

*Corresponding author: Katinka Ónodi-Nagy, Department of Dermatology and Allergology, University of Szeged, Albert Szent-Györgyi Medical Center, 6 Korányi fasor, 6720 Szeged, Hungary, Tel: +36-62-54-52-77; Fax: +36-62-54-59-54; E-mail: onodikatinka@gmail.com

Received date: July 18, 2015; Accepted date: September 01, 2015; Published date: September 08, 2015

Copyright: (c) 2015 Ónodi-Nagy K, et al. This is an open-access article distributed under the terms of the Creative Commons Attribution License, which permits unrestricted use, distribution, and reproduction in any medium, provided the original author and source are credited.

\section{Introduction}

Acute infectious mononucleosis (IM) is mostly caused by a widespread human $\gamma$-herpes virus, the Epstein-Barr virus (EBV) or a human $\beta$-herpes virus, the cytomegalovirus (CMV). Primary infection appears predominantly in children, adolescents and young adults [1]. Symptoms start with a prodromal phase including subfebrility, malaise, arthralgia and myalgia, similar to other common upper respiratory tract infections [2,3]. After the prodromal symptoms, fever, tonsillopharyngitis, cervical lymphadenopathy, leukocytosis and hepatosplenomegaly can develop. The clinical features and a positive heterophil test are usually sufficient to differentiate the condition from bacterial infection and to make the diagnosis of IM [4]. Cutaneous rash may develop during the infection. The incidence of skin eruption in acute IM is 4.2 to $13 \%$ without drug intake [5]. By the frequent use of antibiotics within acute IM, the incidence of skin reactions rises, ranging between $27.8 \%$ and $69 \%$; in some past studies for ampicillin even $90 \%[1,3,6,7]$. According to the literature there is no obvious consensus on the cause of skin symptoms, whether a true drug sensitization or only transient immunoactivation develops.

\section{Materials and Methods}

A comprehensive search was performed in PubMed database from their inception to June 2015. The following expressions were searched without date restriction: "antibiotic rash mononucleosis", "infectious mononucleosis rash", "infectious mononucleosis exanthema", "epstein-barr virus infection", amoxicillin rash viral infection" and "infectious mononucleosis rash histopathology". Results not strictly related to the subject were excluded. Some of the papers were not available to obtain due to their date, language or place of appearance. The suitable articles were then reviewed to identify the possible underlying mechanism of maculopapular rash in patients with infectious mononucleosis following penicillin intake (Figure 1).

\section{Discussion}

\section{Antibiotics: drugs causing rash with the highest incidence within IM}

There are some studies on the usefulness of antiviral, corticosteroid and anaerobic antibacterial (metronidazole) treatments in IM, however the generally accepted therapy is antipyretic and analgetic drugs, enough hydration and rest [4,9-11]. The use of antibiotics is considered mostly unnecessary and is a consequence of misdiagnosis rather than the presence of a concomittant actual bacterial infection [9]. Although patients suffering from IM usually present the classic features, many of them are treated as bacterial infection. In one study it was reported that $90.7 \%$ of IM patients were given antibiotics before hospital admission or serology tests [12]. The incidence of skin rash is higher in the antibiotic treated group than among patients who do not take antibiotics [6,7]. Ampicillin was one of the first drugs which were connected to skin eruptions in IM. However, skin rash was described in connection with other drugs, such as penicillin G or tetracycline, but with much lower incidence [13-15]. Later, other antibacterial drugs were also related to skin symptoms in IM, such as amoxicillin, talampicillin or methicillin [2, 16-18]. A few cases were reported about cephalexin, levofloxacin, erythromycin and azithromycin [5,19-22]. More recent publications, not only case studies but clinical reports on larger patient groups, and reviews on previously published cases, discuss the connection between the viral infection and the antibiotic therapy. In Tables 1 and 2 we summarize literature data on skin rash incidence in IM in connection with various antibacterial therapies.

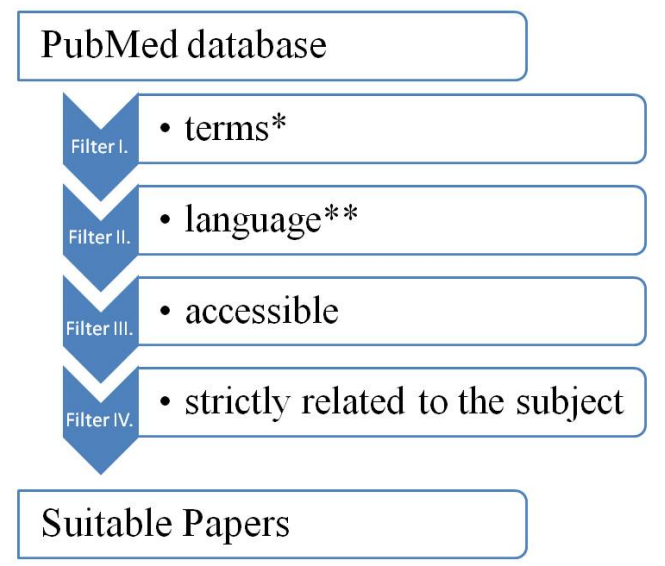

Figure 1: Strategy of database search.

We also included data on our patients with infectious mononucleosis who were treated between 2002 and 2012 at the Department of Dermatology and Allergology, University of Szeged in Hungary [8]. 


\begin{tabular}{|l|l|l|l|l|l|}
\hline Author (s) & Patel [13] & Pullen et al. [52] & Brown et al. [14] & Timár et al. [7] & Chovel-S. et al. [1] \\
\hline Year & 1967 & 1967 & 1967 & 1987 & 2013 \\
\hline Patients & 38 & 184 & 150 & 156 & 238 \\
\hline Range of Age & no statement & $15-24$ years & university students & 7 months-5 years & $0-18$ years \\
\hline Female/Male & no statement & $101 / 83$ & no statement & $70 / 86$ & $132 / 106$ \\
\hline Total rate of skin rash & $42.1 \%(16)$ & $35 \%(64)$ & $30 \%(45)$ & $33.3 \%(52)$ & $30.3 \%(72)$ \\
\hline $\begin{array}{l}\text { Skin rash in patients treated } \\
\text { with antibiotics }\end{array}$ & $55.6 \%(15 / 27)$ & $45 \%(54 / 121)$ & $33.3 \%(42 / 126)$ & $37.7 \%(49 / 130)$ & $32.9 \%(57 / 173)$ \\
\hline $\begin{array}{l}\text { Skin rash in patients } \\
\text { without antibiotics }\end{array}$ & $9 \%(1 / 11)$ & $16 \%(10 / 63)$ & $13 \%(3 / 24)$ & $11.5 \%(3 / 26)$ & $23.1 \%(15 / 65)$ \\
\hline
\end{tabular}

Table 1: Studies of maculopapular rash in infectious mononucleosis.

\begin{tabular}{|c|c|c|c|c|c|}
\hline Author (s) & Patel [13] & Pullen et al. [52] & Brown et al. [14] & Timár et al. [7] & Chovel-S. et al. [1] \\
\hline Year & 1967 & 1967 & 1967 & 1987 & 2013 \\
\hline Ampicillin & $100 \%(13 / 13)$ & $95 \%(18 / 19)$ & $69 \%(20 / 29)$ & $83.8 \%(31 / 37)$ & - \\
\hline Amoxicillin & - & - & - & - & $29.51 \%(18 / 61)$ \\
\hline Amoxicillin+clavulanate & - & - & - & - & $15.56 \%(7 / 45)$ \\
\hline Cephalosporins & - & - & - & $83.3 \%(5 / 6)$ & $15.38 \%(10 / 65)$ \\
\hline Macrolides & - & - & - & $0 \%(0 / 5)$ & $9.09 \%(3 / 33)$ \\
\hline Penicillin & - & $43 \%$ & - & $10.2 \%(9 / 88)$ & $8.57 \% 3 / 35)$ \\
\hline Tetracycline & - & $12 \%(2 / 17)$ & - & $11.5 \%(3 / 26)$ & - \\
\hline Sulfonamid & - & - & - & $11.8 \%(4 / 34)$ & - \\
\hline $\begin{array}{l}\text { Penicillin G or Tetracycline or } \\
\text { Sodium Cloxacillin }\end{array}$ & $14 \%(2 / 14)$ & - & - & - & - \\
\hline
\end{tabular}

Table 2: Incidence of antibacterial drug induced skin rash according to antibiotic type.

\section{An age dependent phenomenon?}

Primary EBV infection appears predominantly in children, adolescents and young adults. The infection occurring before the age of 4 is considered to be asymptomatic or to resemble a nonspecific viral disease, while in adolescents and adults the classical features of the illness are prominent $[1,23]$. Publications on skin rash following IM with and/or without concomitant antibiotic therapy in the age group of few months-babies to young adults usually focus on the role of virus-drug interaction. Only few studies discuss the possible age related differences in the underlying pathomechanism of IM related skin rash. Chovel-Sella et al. couldn't find any association with the age in the development of rash after antibiotic exposure [1]. The immune system of a few months old child is different from an adult, which may play an important role in the development of drug sensitization. It is possible that true drug sensitization occurs with much lower frequency in children, than in young adults. Further investigations are needed to answer whether the mechanism of maculopapular eruptions following antibiotic administration in IM differs in the different age groups.

\section{Clinical characteristics and clinicopathologic findings in IM related rash}

As already stated, primary EBV infection occurring before the age of 4 considered to be asymptomatic or to resemble a nonspecific viral disease, while in adolescents and adults the previously discussed typical symptoms develop. Mucocutaneous involvement occurs in 3\% to $15 \%$ of the patients. The skin eruptions are mostly morbilliform, presented on the trunk, on upper extremities and on the face, after an incubation period, usually lasting up to 7 days. Less common clinical symptoms were also described such as erythema multiforme-like and herpetiform lesions, urticaria, petechiae, transient cold urticaria, immune-related thrombocytopenic purpura, erythema nodosum or pedunculated papule on the tongue. Palatal petechiae or bilateral upper eyelid edema can be helpful diagnostically [23-25]. Studies in the literature report on the clinical evaluation of the skin rash and rarely on skin biopsy.

The concomitant use of antibiotics during infectious mononucleosis increases the occurrence of skin eruptions. In patients taking antibiotics the eruptions usually occurs 2-10 days after starting the antibiotic treatment [26]. The incidence ranges between $27.8 \%$ and 
Page 3 of 6

$69 \%$. Chovel-Sella et al. found the aminopenicillin rash in children is significantly lower than the $90 \%$ incidence rate reported in earlier studies $[1,3,6,7]$. The skin eruptions are mostly diffuse, symmetric maculopapular exanthems on the whole body (Figure 2). Not only morbilliform lesions, but in some cases urticarial, purpuric and vesicular rashes, pustular eyrthematous rash, universal erythema or cutaneous vasculitis in the erythema multiforme pattern were also reported in connection with antibiotic use [16,17,20,27]. Severe cutaneous reactions such as erythema multiforme or Stevens-Johnson syndrome may be possible manifestations [28,29]. Although these cases are well-documented clinically, skin biopsies are rarely taken, and histology reports are exceedingly rare. Because the clinical picture of drug induced skin eruptions appear similar, differentiation by histopathologic examination could be helpful [30]. We took skin biopsies and performed histologic examinations among our patients who developed maculopapular rash following amoxicillin intake in IM (Figure 2). The following histopathologic changes were prominent: hydropic degeneration of basal keratinocytes; mononuclear cells around the vessels and a few eosinophil cells (Figure 3). Sign of leukocytoclasia also appeared in some cases. Both the clinical and histological features confirm a delayed type hypersensitivity reaction [8].

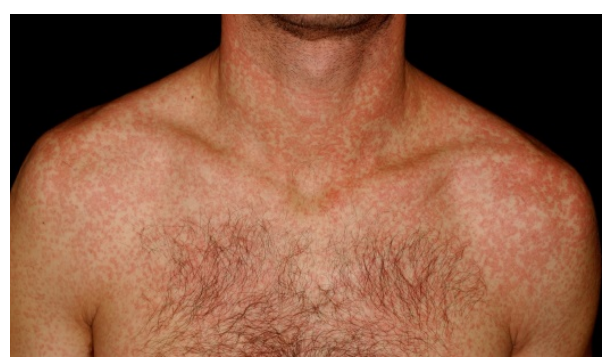

Figure 2: Maculopapular rash in a patient with infectious mononucleosis following amoxicillin intake.

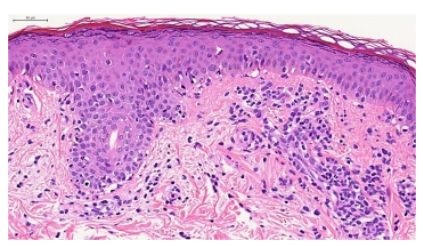

Figure 3: Histolopathologic image of a patient who developed clinically maculopapular rash after amoxicillin exposure.

\section{Pathomechanism: maculopapular rash}

Aminopenicillins may cause immediate hypersensitivity reactions including urticarial, angioedematous or both manifestations and anaphylaxis, however, in IM they are primarily responsible for nonIgE-mediated reactions, such as maculopapular eruptions [31]. Drug induced classic maculopapular drug eruptions are considered to be delayed type hypersensitivity reactions, which involve a Th2-mediated immune response with secretion of IL-4, IL-5 and IL-13 leading to eosinophilic inflammatory reaction. B-cell activation and secretion of IgE and IgG4 antibodies connects the delayed reaction to immediate type I reaction $[26,32]$. Lymphocyte sensitization to $\beta$-lactams can be demonstrated by in vivo and in vitro assays. T-cell proliferative responses can be elicited years after the initial reaction, due to circulating memory T-cells [33].

\section{True hypersensitivity or transient immune reaction?}

Earlier hypothesis: Previously published papers suggest that interactions of viruses and penicillins may predispose individuals to specific illness out-comes. It became an issue whether this phenomenon may lead to persistent, true drug hypersensitivity or it is just a temporary reaction [34].

In the past, in general, it was believed that the morbilliform skin rash following antibiotic intake in patients with IM is a transient reaction, not a true allergic reaction.

Webster et al. proposed the phenomenon to be penicillin specific. In their in vivo and in vitro investigations they aimed to find evidence of specific humoral or cell-mediated immune responses to ampicillin in those patients who developed a rash following antibiotic therapy. They suggested that ampicillin polymer-mediated lymphocyte stimulation may play a role in the development of maculopapular rash. Although, the polymer have weak stimulating effect on lymphocytes, which is probably independent of previous exposure to the drug, they showed a dose dependent widespread lymphocyte stimulation resulting in skin eruptions with altered cell function occurring in IM patients. Antibodies could prevent this reaction. In vivo skin testing proved largely inconclusive, as did investigations of specific penicilloyl antibodies [35].

McKenzie et al. stated that the phenomenon was not true penicillin hypersensitivity, but a non-immunological reaction, because it did not re-appear after the re-administration of the drug. After their investigation of $20 \mathrm{IM}$ (not all of them underwent previous antibiotic therapy) and 20 control patients they concluded that the ampicillin rash in IM resulted from a disseminated reaction of the small blood vessels to circulating ampicillin-antibody complexes. They detected elevated antibody-like activity against ampicillin in both IgM and IgG immunoglobulin classes by means of a sensitive radio-immunoassay. They proposed that the ampicillin induced antibody developed in a similar manner to the Paul-Bunnell antibody, but it was immunologically unrelated. These antibodies occurred without obvious relation to prior ampicillin therapy. They suggested that ampicillin rash could result from interaction of ampicillin with the IgM or IgG antibody to ampicillin generated in the acute phase of infectious mononucleosis. The rash could result as a consequence of complement activation due to circulating antigen-antibody complexes [36,37].

Nazareth et al. also suggested that the sensitivity to ampicillin which develops during IM is not permanent. They applied oral drug provocations months after the onset of skin eruptions. Untoward effects were noted in two of the twenty patients. It was stated that this incidence of $10 \%$ is similar to what appears in the general population [38].

Tímár et al in Hungary investigated the occurrence of drug exanthems among 156 children suffering from IM. There were only 3 cases out of the 26 patients receiving only symptomatic treatment who developed maculopapular exanthems. They found that following ampicillin or cephalexin treatment $83 \%$ of the patients developed skin rash. Exanthems were morbilliform, maculopapular skin eruptions, all appeared about 8 days after the start of the antibiotic therapy. The incidence of skin reactions were less with erythromycin or 
metronidazole. Based on in vitro tests (chromatin activation of blood lymphocytes detected by polarization microscopy and cytophotometry) and in vivo drug provocation tests $60 \%$ of the patients appeared to have developed drug hypersensitivity. Oral drug provocation tests were performed in 9 of 17 individuals who had ampicillin induced rash within IM. The patients with positive in vitro test results proved to have true drug hypersensitization with oral testing, while in vitro negative patients did not develop any reaction during provocation. The authors stated that the mechanism behind these eruptions could be due to the general, virus induced activity of the immune system. One explanation describes the role of circulating antibodies as a passive, anaphylactic immune reaction. After the patient recovers from IM these heterophil antibodies disappear [7].

Current hypothesis: It is generally accepted that viral infections enhance the risk of developing drug allergy. The underlying mechanism is still not fully understood [34]. If within the viral infection the antibiotic therapy induces a lasting drug specific sensitization to the drug, that can be proved later with repeated drug exposure. In these cases both major and minor penicillin antigen determinants could cause the skin symptoms [7].

With changing antibiotic use physicians recognized more cases of skin rash with other antibacterial drugs, such as levofloxacin or azithromycin, in acute IM patients. These cases enforce the notion that there may be no safe antibiotic to prescribe in IM. A possible explanation of transient immune reactions may be that the virus induces a loss of tolerance state that results in transient hypersensitivity, in a transient Th1 lymphocyte-mediated delayed type hypersensitivity reaction to medication. In EBV infection levels of abnormal circulating Th1 lymphocytes are increased, leading to activation of cell-mediated immunity. Th1 lineage mostly produce type 2 interleukin (IL-2), interferon-gamma (INF- $\gamma$ ) and tumor necrosis factor-alpha (TNF- $\alpha$ ). IL-2 is responsible for the stimulation of T-lymphocytes' growth and TNF- $\alpha$ has pro-inflammatory effect. INF- $\gamma$ inhibits the function of Th2 lymphocytes, and their interleukin secretion. Down-regulation of type 4 interleukin (IL-4) decreases B lymphocytes' growth and development. Due to the depression of type 10 interleukin (IL-10) secretion its effect on Th1-lymphocyte suppression is absent $[5,19]$. Case reports, such as a case report by Leung et al. support this concept, as some patients, similar to the one mentioned in this particular article, can take amoxicillin again after the previous rash without developing skin eruptions [2].

Renn et al suggested that the development of true drug hypersensitivity allergic reaction for aminopenicillin during a florid viral infection is definitely more prevalent as it was believed previously. They demonstrated true sensitizations to amoxicillin in three patients with IM. Their patients took amoxicillin during acute IM, they showed positive proliferative responses in the lymphocyte transformation test. In two patient side-chain-specifics sensitization was discovered [6]. Jappe et al provided additional evidence to the development of true delayed type hypersensitivity as well as to the hypothesis of loss of tolerance. Eight patient with primary EBV infection and skin eruption following aminopenicillin intake were examined by in vivo tests. Five of them had positive patch tests to amoxicillin and two of the five to penicillin, in addition. Two of the five had the same reaction years after the first patch test. One patient with positive patch test underwent oral drug challenge and had positive response to amoxicillin and negative to cephalosporin. Another patient of the 5 tolerated the oral penicillin challenge, thereby revealing an isolated allergy to amoxicillin. Two patients were completely negative with all the tests, including drug challenge, in their cases a transient decrease in drug tolerance during EBV infection could explain the skin symptoms [39]. We also reported evidence for the development of true drug allergy during IM. Among our examined ten patients lymphocyte transformation test showed negative results with amoxicillin, while one patient had positive reaction to cefixime. Six patients with suspected sensitization to amoxicillin were then investigated by in vivo tests. Prick tests were negative in all six patients, but the intradermal tests showed positive reactions in four patients [8].

All these studies emphasize the importance of in vivo and in vitro drug hypersensitivity examinations to verify whether true antibiotic sensitization developed within IM. Testing is essential to decide whether individual patients could use the suspected drug in the future or avoidance is necessary. Based on the available clinical data it is safe to say that even in connection with drugs different mechanisms act behind the skin symptoms in IM.

The role of previous and/or present, acute or chronic viral infection, in the development of drug induced reactions has received more attention recently [40]. Molecular mimicry between amoxicillin and viral molecules, or high affinity of betalactams for viral proteins cannot be excluded as potential mechanisms for skin reactions in some patients. Viral infection may be a co-activating factor for drug specific $\mathrm{T}$ cells and without the acute viral infection these drug specific $\mathrm{T}$ cells could apoptose, therefore we could not detect a long lasting hypersensitivity in these patients. It is recognized that Human Herpesvirus 6 (HHV-6), EBV and CMV reactivation appears in drug reaction with eosinophilia and systemic symptoms (DRESS) syndrome or in other severe cutaneous adverse drug reactions, resulting in a marked deviation in the pathological phenotype of severe drug eruptions [40-42]. Mardivirin et al. investigated the influence of amoxicillin on HHV-6 replication by in vitro methods. Retrospectively analyzed amoxicillin flare was observed in 7 patients with DRESS syndrome induced by different drugs. They proposed that amoxicillin induced a flare of DRESS syndrome by acting directly on herpesvirus replication [41]. Although, in patients with acute infectious mononucleosis the maculopapular rash always appears following the antibiotic therapy, there may be similarities in the mechanisms by which the virus and the drug interacts. Due to impaired drug detoxication mechanisms (low plasma glutathione level), drug hypersensitivity reactions are more likely to develop in patients suffering from human immundeficiency virus (HIV) infection $[43,44]$. Similar mechanism, suppressed detoxification by the viral infection, during EBV infection could also be responsible for the developing skin reaction.

\section{The role of in vitro investigation}

Clinical history is crucial in the diagnosis of drug hypersensitivity, but the causative drug should be verified by in vivo and in vitro allergy examinations. In vitro tests are harmless, but they do not have $100 \%$ sensitivity or specificity, therefore they are mostly research tools.

From the biological tests radioallergosorbent tests, immunoenzyme assays, enzyme-linked immunosorbent assays, sepharoseradioimmunoassays and flow cytometric basophil activation test are used to measure serum specific IgE and detect specific surface markers with monoclonal antibodies for evaluating immediate reactions [45].

Several in vitro assays have been used to demonstrate lymphocyte sensitization to $\beta$-lactams. Lymphocyte transformation test (LTT) is 
considered to be a useful diagnostic tool to identify drug induced hypersensitivity reactions. Briefly, peripheral blood mononuclear cells from suspected sensitized individuals are exposed to the culprit drug in order to obtain in vitro evidence of lymphocyte proliferation. The overall sensitivity of LTT among allergic patients is found to be $62 \%$, although after analyzed separately, sensitivity was $64.5 \%$ for immediate reactions and $57.9 \%$ for non-immediate immune responses. LTT specificity was reported to be $92.8 \%[33,46,47]$.

\section{The role if in vivo investigation}

The usefulness of skin tests in the examination of drug hypersensitivity reaction depends much on the case history and the well-recognized clinical symptoms. In severe situations, skin testing has to be performed with caution. In non-immediate reactions patch tests and/or late readings of the intradermal tests after 24, 48, 72 and $96 \mathrm{~h}$ are recommended. Specific standardized skin test reagents are commercially available for penicillins. These reagents, including benzyl-penicilloyl poly-L-lysine, penicilloyl poly-L-lysine, minor determinant mix, amoxicillin and ampicillin from a few companies, like Allergopharma, Beecham, Antibiotic SA or Diater Laboratorios [48-50]. Manufacturers have their own investigation protocols. In general, examination starts by skin prick tests. If responses are negative, intradermal, and then patch tests need to be performed. Luque et al. found that skin tests with $\beta$-lactams were positive in $36,8 \%$ of the patients with nonimmediate reactions [33]. Based on the literature, delayed-reading intradermal tests appear to be slightly more sensitive than patch tests although perhaps less specific [50]. Though skin tests are quite useful in the diagnosis of drug allergy, if possible, and if required, negative cutaneous tests should be confirmed by drug provocation tests in order to prove true drug sensitization [51,52].

\section{Conclusion}

Acute infectious mononucleosis is a relatively frequent illness among young individuals. Besides the classical clinical characteristics generalized maculopapular eruptions may occur among these patients. The incidence of cutaneous rash is significantly higher among patients taking one or more antibacterial drugs during the florid viral infection. The main viewpoint of nearly all previous literature is that an interaction, explained by several hypotheses, exists between the drug and the virus. However, recently published papers indicate that the immune reaction, previously thought to be principally a transient phenomenon, may cause persistent delayed-type hypersensitivity reactions to antibiotics. These data underline the importance of allergy examinations among IM patients who develop skin symptoms while taking antibiotics.

\section{Acknowledgment}

We thank Andrea Gyimesi for her assistance in the preparation of the manuscript. The work was supported by TÁMOP - 4.2.2.A-11/1/ KONV-2012-0035 project.

\section{References}

1. Chovel-Sella A, Ben Tov A, Lahav E, Mor O, Rudich H, et al. (2013) Incidence of rash after amoxicillin treatment in children with infectious mononucleosis. Pediatrics 131: e1424-1427.

2. Leung AK, Rafaat M (2003) Eruption associated with amoxicillin in a patient with infectious mononucleosis. Int J Dermatol 42: 553-555.
3. Treffel P, Gabard B (1996) Measurement of sodium lauryl sulfateinduced skin irritation. Acta Derm Venereol 76: 341-343.

4. Balfour HH Jr, Dunmire SK, Hogquist KA (2015) Infectious mononucleosis. Clin Transl Immunology 4: e33.

5. Dakdouki GK1, Obeid KH, Kanj SS (2002) Azithromycin-induced rash in infectious mononucleosis. Scand J Infect Dis 34: 939-941.

6. Renn CN, Straff W, Dorfmüller A, Al-Masaoudi T, Merk HF, et al. (2002) Amoxicillin-induced exanthema in young adults with infectious mononucleosis: demonstration of drug-specific lymphocyte reactivity. $\mathrm{Br}$ J Dermatol 147: 1166-1170.

7. Timár L, Baló-Banga JM, Budai J (1987) [Infectious mononucleosis and drug exanthema]. Orv Hetil 128: 1871-1874.

8. Ónodi-Nagy K, Kinyó Á, Meszes A, Garaczi E, Kemény L, et al. (2015) Amoxicillin rash in patients with infectious mononucleosis: evidence of true drug sensitization. Allergy Asthma Clin Immunol 11: 1.

9. Lennon P, Crotty M, Fenton JE (2015) Infectious mononucleosis. BMJ 350: h1825.

10. Odumade OA, Hogquist KA, Balfour HH Jr (2011) Progress and problems in understanding and managing primary Epstein-Barr virus infections. Clin Microbiol Rev 24: 193-209.

11. Luzuriaga K, Sullivan JL (2010) Infectious mononucleosis. N Engl J Med 362: 1993-2000.

12. Almási I, Ternák G, Bali I (2001) [Clinical aspects of the diagnosis and treatment of infectious mononucleosis in primary care and in departments of infectious diseases]. Orv Hetil 142: 899-903.

13. Patel BM (1967) Skin rash with infectious mononucleosis and ampicillin. Pediatrics 40: 910-911.

14. Brown GL, Kanwar BS (1967) Drug rashes in glandular fever. Lancet 2: 1418.

15. Nazareth IJ (1971) Ampicillin and mononucleosis. Br Med J 3: 48

16. Mulroy R (1973) Amoxycillin rash in infectious mononucleosis. Br Med J 1: 554 .

17. Copeman PW, Scrivener R (1977) Amoxycillin rash. Br Med J 1: 1354.

18. Fields DA (1980) Methicillin rash in infectious mononucleosis. West J Med 133: 521.

19. Paily R (2000) Quinolone drug rash in a patient with infectious mononucleosis. J Dermatol 27: 405-406.

20. McCloskey GL, Massa MC (1997) Cephalexin rash in infectious mononucleosis. Cutis 59: 251-254.

21. Schissel DJ, Singer D, David-Bajar K (2000) Azithromycin eruption in infectious mononucleosis: a proposed mechanism of interaction. Cutis 65: 163-166.

22. Banerjee I, Mondal S, Sen S, Tripathi SK, Banerjee G (2014) Azithromycin-induced rash in a patient of infectious mononucleosis - a case report with review of literature. J Clin Diagn Res 8: HD01-02.

23. Hall LD, Eminger LA, Hesterman KS, Heymann WR (2015) Epstein-Barr virus: dermatologic associations and implications: part I. Mucocutaneous manifestations of Epstein-Barr virus and nonmalignant disorders. J Am Acad Dermatol 72: 1-19.

24. Nakai H, Sugata K, Usui C, Asano Y, Yamakita T, et al. (2011) A case of erythema multiforme associated with primary Epstein-Barr virus infection. Pediatr Dermatol 28: 23-25.

25. Gao LW, Xie ZD, Liu YY, Wang Y, Shen KL (2011) Epidemiologic and clinical characteristics of infectious mononucleosis associated with Epstein-Barr virus infection in children in Beijing, China. World J Pediatr 7: 45-49.

26. (2007) Drug Hypersensitivity. Karger, Basel, Freyburg.

27. MALLETT AE (1961) Infectious mononucleosis with jaundice and extensive skin rash. (Report of a case). J Coll Gen Pract 4: 337-340.

28. González-Delgado P, Blanes M, Soriano V, Montoro D, Loeda C, et al. (2006) Erythema multiforme to amoxicillin with concurrent infection by Epstein-Barr virus. Allergol Immunopathol (Madr) 34: 76-78.

29. Zawar V, Chuh A, Sankalecha S (2009) Erythema multiforme-like lesions in the course of infectious mononucleosis. J Dermatol Case Rep 3: 44-46. 
Citation: Ónodi-Nagy K, Bata-Csörgo Z, Varga E, Kemény L, Kinyó A (2015) Antibiotic Induced Cutaneous Rash in Infectious Mononucleosis:

Page 6 of 6

30. Hussein MR (2015) Drug-induced skin reactions: a pathologist viewpoint. Cutan Ocul Toxicol .

31. Romano A, Di Fonso M, Papa G, Pietrantonio F, Federico F, et al. (1995) Evaluation of adverse cutaneous reactions to aminopenicillins with emphasis on those manifested by maculopapular rashes. Allergy 50: 113-118.

32. Kuruvilla M, Khan DA (2015) Eosinophilic Drug Allergy. Clin Rev Allergy Immunol .

33. Luque I, Leyva L, José Torres M, Rosal M, Mayorga C, et al. (2001) In vitro T-cell responses to beta-lactam drugs in immediate and nonimmediate allergic reactions. Allergy 56: 611-618.

34. Haverkos HW, Amsel Z, Drotman DP (1991) Adverse virus-drug interactions. Rev Infect Dis 13: 697-704.

35. Webster AW, Thompson RA (1974) The ampicillin rash. Lymphocyte transformation by ampicillin polymer. Clin Exp Immunol 18: 553-564.

36. McKenzie H, Parratt D, White RG (1976) IgM and IgG antibody levels to ampicillin in patients with infectious mononucleosis. Clin Exp Immunol 26: 214-221.

37. Wands JR, Perrotto JL, Isselbacher KJ (1976) Circulating immune complexes and complement sequence activation in infectious mononucleosis. Am J Med 60: 269-272.

38. Nazareth I, Mortimer P, McKendrick GD (1972) Ampicillin sensitivity in infectious mononucleosis--temporary or permanent? Scand J Infect Dis 4: 229-230.

39. Jappe U (2007) Amoxicillin-induced exanthema in patients with infectious mononucleosis: allergy or transient immunostimulation? Allergy 62: 1474-1475.

40. Hirahara K, Kano Y, Mitsuyama Y, Takahashi R, Kimishima M, et al (2010) Differences in immunological alterations and underlying viral infections in two well-defined severe drug eruptions. Clin Exp Dermatol 35: 863-868.

41. Mardivirin L, Valeyrie-Allanore L, Branlant-Redon E, Beneton N, Jidar $\mathrm{K}$, et al. (2010) Amoxicillin-induced flare in patients with DRESS (Drug Reaction with Eosinophilia and Systemic Symptoms): report of seven cases and demonstration of a direct effect of amoxicillin on Human Herpesvirus 6 replication in vitro. Eur J Dermatol 20: 68-73.
42. Chen YC, Chiang HH, Cho YT, Chang CY, Chen KL, et al. (2015) Human herpes virus reactivations and dynamic cytokine profiles in patients with cutaneous adverse drug reactions - a prospective comparative study. Allergy 70: 568-575.

43. Para MF, Finkelstein D, Becker S, Dohn M, Walawander A, et al. (2000) Reduced toxicity with gradual initiation of trimethoprimsulfamethoxazole as primary prophylaxis for Pneumocystis carinii pneumonia: AIDS Clinical Trials Group 268. J Acquir Immune Defic Syndr 24: 337-343.

44. Smith KJ, Skelton HG, Yeager J, Ledsky R, Ng TH, et al. (1997) Increased drug reactions in HIV-1-positive patients: a possible explanation based on patterns of immune dysregulation seen in HIV-1 disease. The Military Medical Consortium for the Advancement of Retroviral Research (MMCARR). Clin Exp Dermatol 22: 118-123.

45. Romano A, Demoly P (2007) Recent advances in the diagnosis of drug allergy. Curr Opin Allergy Clin Immunol 7: 299-303.

46. Pichler WJ, Tilch J (2004) The lymphocyte transformation test in the diagnosis of drug hypersensitivity. Allergy 59: 809-820.

47. Nyfeler B, Pichler WJ (1997) The lymphocyte transformation test for the diagnosis of drug allergy: sensitivity and specificity. Clin Exp Allergy 27: 175-181.

48. Brockow K, Romano A, Blanca M, Ring J, Pichler W, et al. (2002) General considerations for skin test procedures in the diagnosis of drug hypersensitivity. Allergy 57: 45-51.

49. Romano A, Viola M, Bousquet PJ, Gaeta F, Valluzzi R, et al. (2007) A comparison of the performance of two penicillin reagent kits in the diagnosis of beta-lactam hypersensitivity. Allergy 62: 53-58.

50. Romano A, Viola M, Gaeta F, Rumi G, Maggioletti M (2008) Patch testing in non-immediate drug eruptions. Allergy Asthma Clin Immunol 4: 66-74.

51. Demoly P, Adkinson NF, Brockow K, Castells M, Chiriac AM, et al. (2014) International Consensus on drug allergy. Allergy 69: 420-437.

52. Pullen H, Wright N, Murdoch JM (1967) Hypersensitivity reactions to antibacterial drugs in infectious mononucleosis. Lancet 2: 1176-1178. 